\title{
Impact of Hydroxyurea on Survival and Risk of Thrombosis Among Older Patients With Essential Thrombocythemia
}

\author{
Nikolai A. Podoltsev, MD, PhDa; Mengxin Zhu, MPH',c; Amer M. Zeidan, MBBSa; Rong Wang, PhD b,c; \\ Xiaoyi Wang, $\mathrm{MPH}^{\mathrm{b}, \mathrm{c}}$; Amy J. Davidoff, $\mathrm{PhD}^{\mathrm{c}, \mathrm{d}}$; Scott F. Huntington, MD, MPH ${ }^{\mathrm{a}, \mathrm{c}}$; Smith Giri, MBBSa; \\ Steven D. Gore, MD; and Xiaomei $\mathrm{Ma}, \mathrm{PhD}^{\mathrm{b}, \mathrm{c}}$
}

\begin{abstract}
Background: Current guidelines recommend hydroxyurea (HU) as frontline therapy for patients with high-risk essential thrombocythemia (ET) to prevent thrombosis. However, little is known about the impact of $\mathrm{HU}$ on thrombosis or survival among these patients in the real-world setting. Patients and Methods: A retrospective cohort study was conducted of older adults (aged $\geq 66$ years) diagnosed with ET from 2007 through 2013 using the linked SEERMedicare database. Multivariable Cox proportional hazards regression models were used to assess the effect of HU on overall survival, and multivariable competing risk models were used to assess the effect of $\mathrm{HU}$ on the occurrence of thrombotic events. Results: Of 1,010 patients, 745 (73.8\%) received $\mathrm{HU}$. Treatment with $\mathrm{HU}$ was associated with a significantly lower risk of death (hazard ratio [HR], 0.52; $95 \% \mathrm{Cl}$, $0.43-0.64 ; P<.01$ ). Every $10 \%$ increase in $\mathrm{HU}$ proportion of days covered was associated with a $12 \%$ decreased risk of death $(H R, 0.88$; $95 \% \mathrm{Cl}, 0.86-0.91 ; \mathrm{P}<.01)$. Compared with nonusers, $\mathrm{HU}$ users also had a significantly lower risk of thrombotic events $(\mathrm{HR}, 0.51 ; 95 \% \mathrm{Cl}$, $0.41-0.64 ; P<.01)$. Conclusions: Although underused in our study population, $\mathrm{HU}$ was associated with a reduced incidence of thrombotic events and improved overall survival in older patients with ET.
\end{abstract}

J Natl Compr Canc Netw 2019;17(3):211-219 doi: 10.6004/jnccn.2018.7095

aDepartment of Internal Medicine, Section of Hematology, Yale School of Medicine; ${ }^{b}$ Department of Chronic Disease Epidemiology, Yale School of Public Health; 'Yale Cancer Outcomes, Public Policy, and Effectiveness Research Center; and d Department of Health Policy and Management, Yale School of Public Health, New Haven, Connecticut.

\section{Background}

Essential thrombocythemia (ET) is a clonal myeloproliferative neoplasm (MPN) characterized by persistently elevated platelet counts. The median age at diagnosis is 68 years $^{1}$ and median survival is 19.8 years, which is inferior to that of the age- and sex-matched US population. ${ }^{2}$ Patients with ET are at $>20 \%$ risk for thrombotic complications. ${ }^{3,4}$ Thus, a major goal of ET treatment is thrombosis prevention. Patients are categorized into high- and low-risk groups based on the presence of advanced age ( $>60$ years) or thrombosis history. ${ }^{5}$ Both NCCN and the European LeukemiaNet (ELN) guidelines recommend cytoreductive therapy with hydroxyurea (HU) as frontline therapy for patients with high-risk ET. ${ }^{6,7}$

$\mathrm{HU}$ is an oral chemotherapeutic agent that inhibits ribonucleotide reductase and interferes with the process of DNA synthesis and repair. ${ }^{8}$ Three randomized trials have evaluated the effect of $\mathrm{HU}$ among patients with high-risk ET. ${ }^{9-11}$ The Bergamo study found HU therapy to be superior in lowering platelet counts and reducing thrombotic complications compared with no cytoreductive therapy. ${ }^{9}$ In the UK-PT1 study, HU was superior to anagrelide, a selective platelet-lowering agent, in reducing arterial thrombosis, major bleeding, and fibrotic progression. However, it showed that anagrelide was superior in preventing venous thrombosis. ${ }^{10}$ The more recent ANAHYDRET study enrolled patients with high-risk ET using the WHO-defined diagnostic criteria and reported that $\mathrm{HU}$ and anagrelide were similar in the prevention of thrombotic end points. ${ }^{11}$ Current guidelines recommend anagrelide (if not previously used as initial therapy) as a second-line treatment for patients refractory to or intolerant of HU.,7

To date, the impact of HU on thrombosis and survival among patients with high-risk ET has rarely been evaluated outside of clinical trials, and some experts have

See JNCCN.org for supplemental online content. 
expressed concern about the safety and efficacy of HU in the real-world setting. ${ }^{12}$ To address these knowledge gaps, a large, population-based, nationally representative cohort study was conducted to assess the impact of HU on survival and thrombosis risk among older patients with ET.

\section{Patients and Methods}

\section{Data Source}

The linked SEER-Medicare database, developed by the NCI and the Centers for Medicare \& Medicaid Services, links patient-level information on incident cancer diagnoses from SEER registries to a master file of Medicare enrollment and claims for inpatient, outpatient, physician services, and prescription drugs. ${ }^{13}$ The SEER registries are nationally representative and account for approximately $28 \%$ of the US population. ${ }^{14}$ ET has been reported to SEER registries since 2001, providing a unique opportunity to access a representative sample of patients with ET. Because most patients with ET are diagnosed after 65 years of age, a large number of patients with ET were able to be ascertained using the SEER-Medicare database. ${ }^{15,16}$ The Yale Human Investigation Committee determined that this study did not directly involve human subjects.

\section{Patient Cohort}

We identified patients who were diagnosed with ET (ICDO-3 code: 9962 from SEER records) from 2007 through 2013. To avoid curtailing the observation time caused by reporting delay from SEER, ${ }^{17}$ we searched Medicare claims within 1 year before the SEER-reported date of ET diagnosis. If a patient had an ICD-9 code indicative of ET (238.71) from 1 inpatient claim or at least 2 outpatient claims, which were $>30$ days apart, the date of the earliest ET claim, instead of the SEER-reported date of diagnosis, was used as the diagnosis date. All patients were required to: (1) be aged 66 (because of the need to obtain Medicare claims for 1 year before diagnosis to calculate comorbidity scores and other covariates) to 99 years (to avoid potential changes in the pattern of care for those aged $\geq 100$ years $^{18,19}$ ) at diagnosis; (2) have known month of diagnosis; (3) not be reported from autopsy or death certificate only; (4) have continuous enrollment in Medicare Parts A and B from 1 year before diagnosis to the end of follow-up; (5) have continuous Part D coverage from diagnosis to the end of follow-up; and (6) have survived at least 30 days after ET diagnosis. All patients were followed to death or end of study (December 31, 2014), whichever occurred first.

\section{HU Use}

HU use after ET diagnosis was assessed by searching Medicare Part D claims from diagnosis through the end of follow-up. In addition, HU use was measured by calculating the proportion of days covered (PDC) by HU prescriptions from ET diagnosis through the end of follow-up; PDC is commonly used to describe patients' adherence to medications. ${ }^{20}$ In our analysis, a patient who never received HU would have a PDC of $0 \%$, and a patient who had taken HU every day from ET diagnosis through the end of follow-up would have a PDC of $100 \%$.

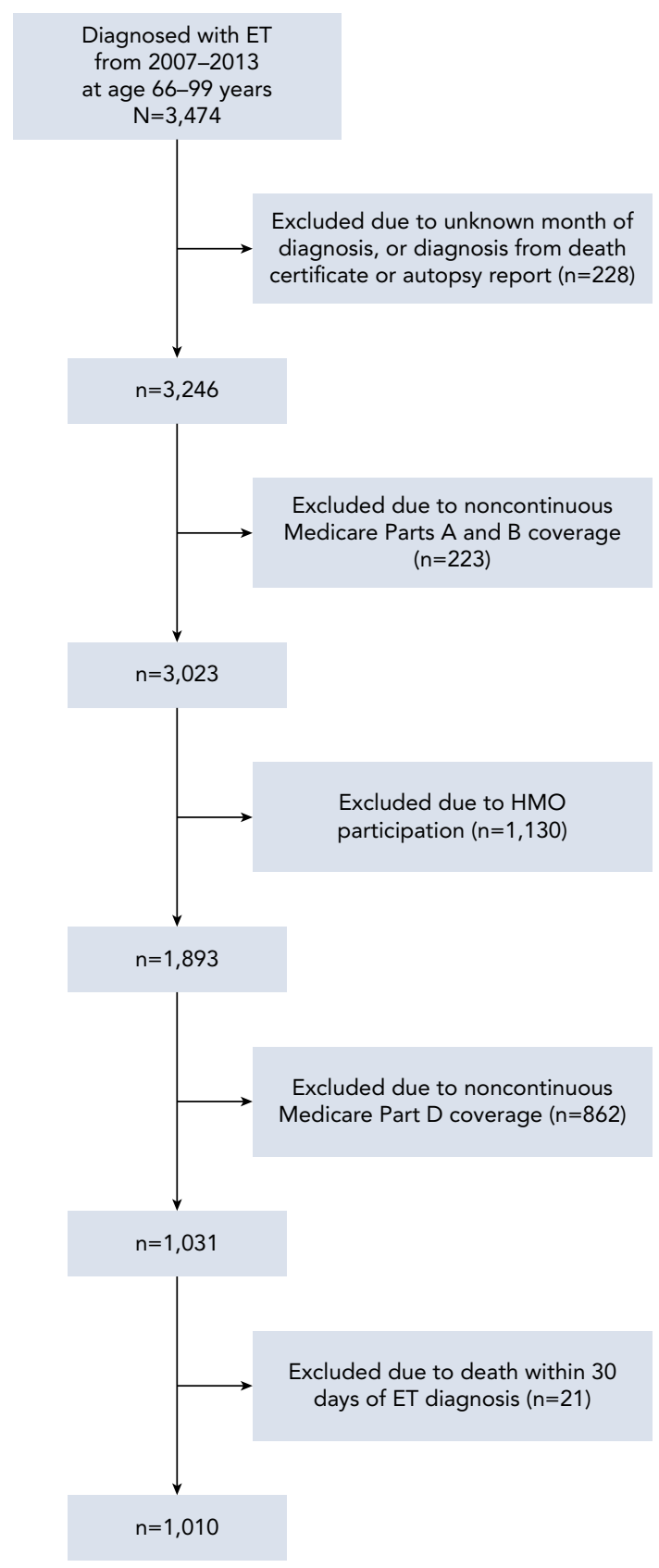

Figure 1. Construction of the study cohort.

Abbreviation: $E T$, essential thrombocytopenia. 


\section{Outcomes}

We were interested in both overall survival and the first occurrence of thrombotic events after ET diagnosis, including venous thrombosis (deep vein thrombosis, pulmonary embolism, superficial thrombophlebitis), arterial thrombosis (stroke, transient ischemic attack, angina, acute myocardial infarction/acute coronary syndrome, arterial embolism, peripheral arterial thrombosis), and sudden death. We identified these thrombotic events by adapting a previously used claims-based algorithm ${ }^{21}$ (see supplemental eAppendix 1, available with this article at JNCCN.org).

\section{Other Variables of Interest}

We obtained information on the following sociodemographic characteristics: age at diagnosis, sex, race, and Medicare Part D low-income subsidy, a marker for reduced out-of-pocket cost sharing and lower socioeconomic status.
Receipt of influenza vaccination in the 12 months before ET diagnosis was included in our multivariable analysis as an indicator of access to the healthcare system, ${ }^{21}$ because this may influence treatments or outcomes. Because performance status is an important factor in clinical decision-making, each patient's disability status ${ }^{22}$ was evaluated as a proxy of poor performance status before diagnosis. To assess comorbidity, we searched for ICD-9 diagnosis codes in the 12 months before ET diagnosis that appeared on any inpatient claims or at least 2 outpatient claims $>30$ days apart, ${ }^{23}$ and then developed a modified Elixhauser score by removing prior thrombosis from the original Elixhauser score. Prior thrombosis within the 12 months before ET diagnosis was ascertained by adapting the algorithm developed by Gupta et $\mathrm{al}^{21}$ (supplemental eAppendix 1). With the modified Elixhauser score and the history of thrombosis, all comorbidities were captured.

\section{Table 1. Patient Characteristics}

\begin{tabular}{|c|c|c|c|c|}
\hline & $\begin{array}{c}\text { Overall }(N=1,010) \\
n(\%)\end{array}$ & $\begin{array}{c}\text { HU Users }(\mathrm{N}=745) \\
n(\%)\end{array}$ & $\begin{array}{c}\text { HU Nonusers ( } \mathbf{N}=265) \text {, } \\
n(\%)\end{array}$ & $P$ Value ${ }^{a}$ \\
\hline Median age, y (IQR) & 79 (73-84) & $78(72-84)$ & 79 (73-85) & .25 \\
\hline Female & $685(67.8)$ & $510(68.5)$ & $175(66.0)$ & \\
\hline Male & $325(32.2)$ & 235 (31.5) & $90(34.0)$ & \\
\hline Nonwhite & $137(13.6)$ & $91(12.2)$ & 46 (17.4) & \\
\hline Modified Elixhauser score & & & & $<.01$ \\
\hline 0 & $366(36.2)$ & $302(40.5)$ & $64(24.2)$ & \\
\hline 1 & $256(25.4)$ & $199(26.7)$ & $57(21.5)$ & \\
\hline Yes & $150(14.8)$ & $106(14.2)$ & $44(16.6)$ & \\
\hline Disability & & & & $<.01$ \\
\hline No & $845(83.7)$ & $651(87.4)$ & $194(73.2)$ & \\
\hline Yes & $165(16.3)$ & $94(12.6)$ & $71(26.8)$ & \\
\hline Low-income subsidy & & & & $<.01$ \\
\hline No & $729(72.2)$ & $567(76.1)$ & $162(61.1)$ & \\
\hline Yes & $281(27.8)$ & $178(23.9)$ & $103(38.9)$ & \\
\hline Influenza vaccination withir & fore ET diagnosis & & & .49 \\
\hline No & $405(40.1)$ & $294(39.5)$ & $111(41.9)$ & \\
\hline
\end{tabular}

Abbreviations: $\mathrm{ET}$, essential thrombocythemia; $\mathrm{HU}$, hydroxyurea; IQR, interquartile range.

a ${ }^{a}$ hi-square test $P$ value for categorical covariates and $t$ test $P$ value for continuous covariates. 
Use of cytoreductive agents other than HU, including anagrelide, interferons, busulfan, and ruxolitinib, was also ascertained by searching Medicare Parts B and D claims from diagnosis through end of follow-up.

\section{Statistical Analysis}

Categorical variables were presented using frequencies and percentages, and continuous variables were summarized by median and interquartile range (IQR). Consistent with the SEER-Medicare requirement to preserve confidentiality, all categories with $\leq 10$ patients were reported as $<11$. Characteristics of patients who fulfilled the eligibility criteria and were included in the study were compared with those who were excluded from the study using chi-square tests for categorical variables (eg, race) and $t$ tests for continuous variables (eg, age).

For patients included in the study, baseline characteristics of the patients by HU use were compared using chi-square test for categorical variables and $t$ test for continuous variables. Kaplan-Meier curves and logrank tests were used to compare overall survival and the incidence of thrombotic events between patient groups. For both outcomes, patients were followed from the date of diagnosis to the relevant end point or end of the study period (December 31, 2014), whichever came first.

Multivariable Cox proportional hazards regression models were used to examine the effect of HU/HU PDC on survival. Multivariable competing risk regression models were used to examine the effect of HU/HU PDC on thrombosis after diagnosis, with death as the competing risk. The assumption of proportional hazard ratios (HRs) was checked using Schoenfeld residuals test. ${ }^{24}$ All multivariable models included age at diagnosis, sex, race, disability status, low-income subsidy, receipt of influenza vaccination in the 12 months before diagnosis, modified Elixhauser

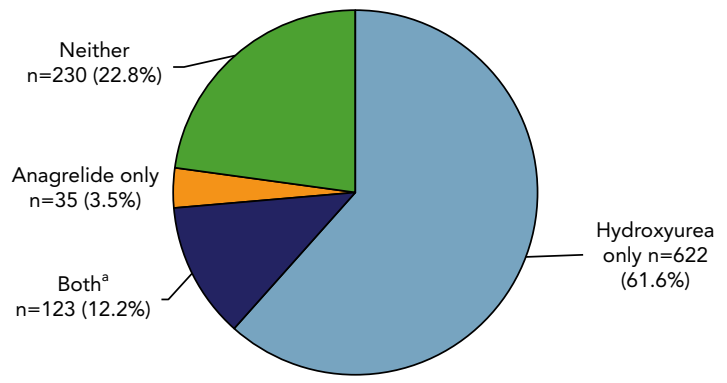

Figure 2. Receipt of hydroxyurea (HU) and anagrelide among 1,010 patients with essential thrombocytopenia.

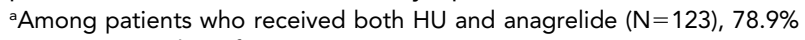
$(n=97)$ received $\mathrm{HU}$ first. comorbidity score, prior thrombosis, and anagrelide use as covariates.

For sensitivity analyses, the analysis with thrombosis as the outcome was performed by excluding patients who had experienced thrombotic events within 30 days of ET diagnosis to avoid detection bias at time of diagnosis. ${ }^{4}$ All patients who ever received any cytoreductive agents other than HU were also excluded to better evaluate the independent impact of $\mathrm{HU}$ on clinical outcomes. Patients who had prior thrombosis were also excluded as a sensitivity analysis.

All analyses were conducted using SAS 9.4 (SAS Institute Inc.) with 2 -sided tests, and a type I error of $5 \%$ as the threshold for statistical significance.

\section{Results}

\section{Patient Characteristics}

A total of 1,010 patients with ET were included in the study (Figure 1). No statistically significant differences were observed between the 1,010 patients who were included in the study and the 2,464 patients who were excluded, in terms of age, sex, and race.

Among 1,010 patients, the median age was 79 years (IQR, 73-84 years), most were female (67.8\%) and white (86.4\%), and median follow-up was 2.83 years (IQR, 1.58-4.60 years) (Table 1).

When we assessed receipt of cytoreductive treatments regardless of the number of claims, we found that $73.8 \%(n=745)$ and $15.6 \%(n=158)$ of all patients received $\mathrm{HU}$ and anagrelide, respectively (Figure 2). Other cytoreductive agents, including interferons $(\mathrm{n}<11)$, busulfan $(\mathrm{n}<11)$, and ruxolitinib $(\mathrm{n}=11)$, were used infrequently and were not further evaluated. Of all patients in our cohort, $22.5 \%(n=227)$ did not receive any of the cytoreductive agents mentioned.

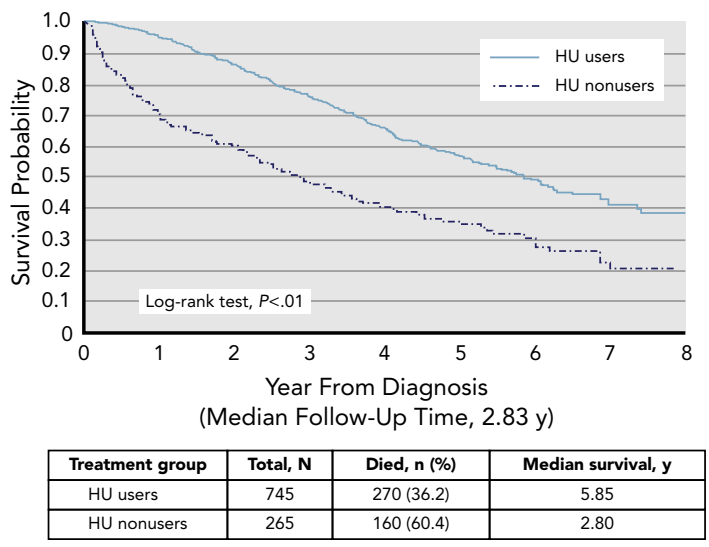

Figure 3. Kaplan-Meier curves for overall survival by hydroxyurea (HU) use. 
Overall Survival

At the end of follow-up, $36.2 \%(n=270)$ of HU users and $60.4 \%(n=160)$ of nonusers died. Median survival was 5.85 and 2.80 years for HU users and nonusers, respectively (log-rank test, $P<.01$; Figure 3 ). In the multivariable Cox proportional hazards analysis, treatment with $\mathrm{HU}$ after diagnosis was associated with a significantly lower risk of death (HR, 0.52; 95\% CI, 0.43-0.64; $P<.01$ ). Every $10 \%$ increase in HU PDC was associated with a $12 \%$ decreased risk of death (HR, 0.88; 95\% CI, 0.86-0.91; $P<.01$ ). Factors associated with inferior survival included advanced age, male sex, presence of comorbidities, poor disability status, and receipt of low-income subsidy. Anagrelide use did not seem to be a significant predictor of survival (Table 2).

\section{Thrombosis After Diagnosis}

A total of 357 patients (35.3\%) developed thrombotic events after diagnosis, including 273 arterial thromboses and 84 venous thromboses and sudden deaths (sudden death $<11$ ). Thrombotic events occurred in $29.2 \%$ $(\mathrm{n}=198)$ of HU users and $47.7 \%(\mathrm{n}=159)$ of HU nonusers. Median time to develop thrombosis was significantly longer among HU users (not computable) compared with nonusers (2.39 years; log-rank test, $P<.01$; Figure 4 ).

\section{Table 2. Multivariable Cox Proportional Hazards Analysis for Overall Survival}

\begin{tabular}{|c|c|c|c|c|c|c|}
\hline Characteristic & \multicolumn{3}{|c|}{ Model 1a } & \multicolumn{3}{|c|}{ Model 2a } \\
\hline \multicolumn{7}{|l|}{ Hydroxyurea use } \\
\hline No & Ref & & & & & \\
\hline Yes & 0.52 & $0.43-0.64$ & $<.01$ & & & \\
\hline Hydroxyurea: PDC (every 10\%) & & & & 0.88 & $0.86-0.91$ & $<.01$ \\
\hline \multicolumn{7}{|l|}{ Sex } \\
\hline Female & Ref & & & Ref & & \\
\hline Male & 1.87 & $1.53-2.30$ & $<.01$ & 1.84 & $1.50-2.26$ & $<.01$ \\
\hline \multicolumn{7}{|l|}{ Race } \\
\hline White & Ref & & & Ref & & \\
\hline 1 & 1.36 & $1.03-1.81$ & .03 & 1.33 & $1.00-1.76$ & .05 \\
\hline$>1$ & 2.12 & $1.64-2.74$ & $<.01$ & 2.08 & $1.61-2.69$ & $<.01$ \\
\hline \multicolumn{7}{|l|}{ History of thrombosis } \\
\hline No & Ref & & & Ref & & \\
\hline Yes & 1.26 & $0.99-1.59$ & .06 & 1.23 & $0.97-1.56$ & .09 \\
\hline \multicolumn{7}{|l|}{ Disability } \\
\hline No & Ref & & & Ref & & \\
\hline Yes & 1.62 & $1.26-2.09$ & $<.01$ & 1.65 & $1.28-2.13$ & $<.01$ \\
\hline \multicolumn{7}{|l|}{ Low-income subsidy } \\
\hline No & Ref & & & Ref & & \\
\hline Yes & 1.03 & $0.80-1.33$ & .83 & 0.83 & $0.64-1.07$ & .14 \\
\hline
\end{tabular}

Abbreviations: HR, hazard ratio; PDC, proportion of days covered.

aAll variables listed under Model 1 were simultaneously included in the same model and mutually adjusted. The same applies to Model 2. 


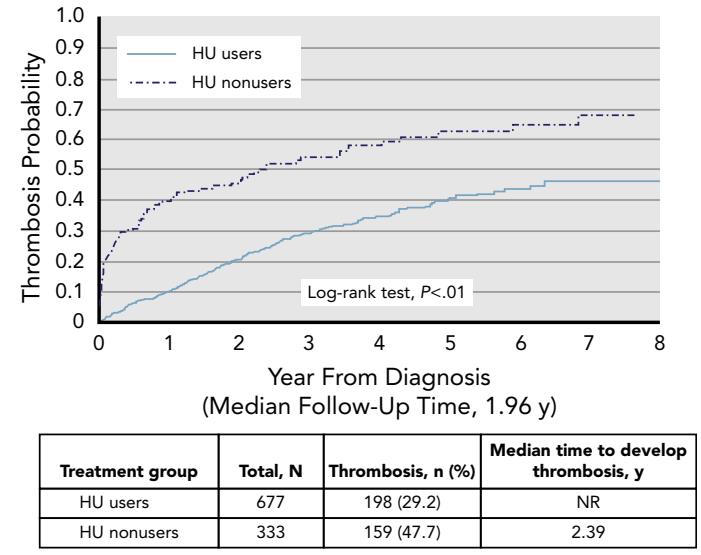

Figure 4. Kaplan-Meier curves for thrombotic events by hydroxyurea (HU) use.

Abbreviation: NR, not reached.

The multivariable competing risk model showed that, compared with nonusers, HU users had a significantly lower risk of developing thrombotic events (HR, 0.51; 95\% CI, 0.41-0.64; $P<.01$; Table 3). Every $10 \%$ increase in HU PDC was not significantly associated with lower risk of thrombosis (HR, 0.99; 95\% CI, $0.96-1.02 ; P=.36$ ). Factors associated with higher risk of thrombosis included advanced age and receipt of low-income subsidy. Anagrelide use was not significantly associated with risk of thrombosis in the model.

\section{Sensitivity Analyses}

To better evaluate the independent impact of HU on clinical outcomes, we conducted sensitivity analyses by excluding patients who received any cytoreductive treatments other than $\mathrm{HU}$ (ie, anagrelide, interferon, busulfan, ruxolitinib) any time between ET diagnosis and the occurrence of death or thrombotic events. The number of patients who remained after these exclusions was 841 and 850 for the analysis of overall survival and thrombosis, respectively. Results from the analyses with these smaller cohort sizes were essentially the same as those observed with the original cohort of 1,010 patients (detailed data not shown). Additionally, excluding patients who experienced thrombosis within 30 days of ET diagnosis (941 patients remaining) or those who had prior thrombosis (860 patients remaining) did not change our results in any meaningful way (detailed data not shown).

\section{Discussion}

In this large, population-based, nationally representative cohort study that reflects contemporary clinical practice in the real-world setting, treatment with HU was associated with improved overall survival in older patients with ET, a finding that, to our knowledge, has not been reported in previous studies. Furthermore, our findings are consistent with results from randomized trials regarding the efficacy of $\mathrm{HU}$ in preventing thrombosis, including those of the Bergamo study, which randomized patients with high-risk ET to HU or no cytoreductive treatment. ${ }^{9}$ Two other trials that compared the effect of HU versus anagrelide drew different conclusions. ${ }^{10,11}$ The UK-PT1 study concluded that HU was superior to anagrelide, ${ }^{10}$ whereas the ANAHYDRET study suggested that the 2 agents were comparable. ${ }^{11}$ In our study, anagrelide was prescribed to $15.7 \%$ patients, of whom $77.8 \%$ were never treated with HU. The relatively small number of patients who received anagrelide limited our ability to directly compare these 2 cytoreductive agents.

The median age of patients with ET included in the UK-PT1 and ANAHYDRET studies ${ }^{10,11}$ was approximately 60 years, which is younger than the median age at diagnosis of 68 years observed at the population level. ${ }^{1}$ Because age is a known risk factor of thrombosis, ${ }^{25}$ it is important to study the effect of $\mathrm{HU}$ in patients with ET in all age groups. From this perspective, our study is particularly informative due to the inclusion of older patients, who account for most patients with ET diagnosed at the population level but who would not have been well represented in clinical trials. In our study, $35 \%$ of patients developed thrombotic events after ET diagnosis, which was higher than what was reported by the UK-PT1 ${ }^{10}$ and ANAHYDRET ${ }^{11}$ studies, and likely attributable to the older median age of our cohort of patients.

In our study of patients with ET diagnosed at age $\geq 66$ years, $>22 \%$ did not receive any cytoreductive agents, which is the treatment indicated for patients at high risk for thrombosis according to the 2-tiered thrombotic risk score. This scoring system is supported by older ELN guidelines, ${ }^{5}$ was used to select patients with high-risk ET for the aforementioned randomized control trials, and continues to be widely adopted in clinical practice. ${ }^{26}$ The NCCN Guidelines for MPNs ${ }^{6}$ and independent MPN experts ${ }^{3}$ favor use of the revised International Prognostic Score of Thrombosis for ET (IPSET-thrombosis), which categorize patients into 4 risk groups based on age $>60$ years, history of thrombosis, and JAK2 V617F mutation positivity. ${ }^{27}$ Cytoreductive therapy is recommended for all patients in the high-risk group (thrombosis history or age $>60$ years with positive $J A K 2$ mutation) and may be indicated for some patients in the intermediate-risk group (age $>60$ years, no thrombosis history, and negative JAK2 mutation). Treatment recommendations for each of the aforementioned risk categories were not examined in the context of prospective controlled studies. We estimate that most patients in our cohort belong to the high-risk group based on the revised IPSET-thrombosis system and 


\begin{tabular}{|c|c|c|c|c|c|c|}
\hline Characteristic & HR & $95 \% \mathrm{Cl}$ & $P$ Value & HR & $95 \% \mathrm{Cl}$ & $P$ Value \\
\hline \multicolumn{7}{|l|}{ Hydroxyurea use ${ }^{a}$} \\
\hline No & Ref & & & & & \\
\hline Age, y & 1.02 & $1.00-1.03$ & .03 & 1.02 & $1.00-1.03$ & .01 \\
\hline \multicolumn{7}{|l|}{ Sex } \\
\hline Female & Ref & & & Ref & & \\
\hline Male & 1.00 & $0.79-1.26$ & .97 & 1.03 & $0.82-1.29$ & .81 \\
\hline \multicolumn{7}{|l|}{ Modified Elixhauser score } \\
\hline 0 & Ref & & & Ref & & \\
\hline 1 & 1.05 & $0.79-1.39$ & .76 & 1.06 & $0.80-1.41$ & .69 \\
\hline$>1$ & 1.19 & $0.89-1.58$ & .24 & 1.33 & $1.01-1.75$ & .04 \\
\hline \multicolumn{7}{|l|}{ History of thrombosis } \\
\hline No & Ref & & & Ref & & \\
\hline Yes & 1.14 & $0.84-1.56$ & .40 & 1.11 & $0.82-1.50$ & .50 \\
\hline \multicolumn{7}{|l|}{ Disability } \\
\hline No & Ref & & & Ref & & \\
\hline Yes & 0.86 & $0.69-1.08$ & .20 & 0.87 & $0.70-1.08$ & .20 \\
\hline \multicolumn{7}{|l|}{ Anagrelide use } \\
\hline No & Ref & & & Ref & & \\
\hline Yes & 1.07 & $0.79-1.44$ & .67 & 1.02 & $0.76-1.38$ & .88 \\
\hline
\end{tabular}

Abbreviations: HR, hazard ratio; PDC, proportion of days covered.

aAll variables listed under Model 1 were simultaneously included in the same model and mutually adjusted. The same applies to Model 2.

have indications for cytoreductive therapy, because all of them were aged $\geq 66$ years, $14.8 \%$ had prior thrombosis, and more than half were expected to be JAK2 mutation-positive. We performed additional analyses by excluding patients with prior history of thrombosis, with results still showing improved survival and decreased thrombotic events among HU users, which favors treatment with $\mathrm{HU}$ for all patients with ET aged $\geq 66$ years.

Our study has several strengths because it investigated a large, population-based cohort of older patients with ET in the United States. The 3 randomized clinical trials of
HU in patients with ET were all conducted in Europe and included younger patients. ${ }^{9-11}$ The nationwide Medicare claims data that we leveraged to conduct this study covered a wide spectrum of health services, regardless of where patients received care, therefore providing comprehensive information on the treatments received by patients. For cytoreductive agents such as HU, we not only had information on use versus nonuse but also could evaluate how many days a patient filled the specific prescription, which allowed us to calculate PDC and investigate a potential dose-response relationship. Furthermore, the linked SEER-Medicare database also 
enabled us to control for other factors that may influence treatment decisions and risk factors for mortality and thrombotic events after diagnosis.

Use of claims data also resulted in some limitations. First, because we relied on Medicare claims to evaluate ET treatments, we were unable to capture agents not covered by Medicare, such as aspirin. Second, we did not have access to any laboratory results, such as platelet and WBC counts, or driver mutation status such as $J A K 2 \mathrm{~V} 617 \mathrm{~F}$, $C A L R$, and MPL mutations. Third, we were unable to assess the impact of therapy on symptoms or the quality of response to HU because this information was not included in the dataset used. Fourth, because use of anagrelide was relatively uncommon in our patient cohort, we did not have the statistical power to compare its effect versus HU. Also, because the development of acute myeloid leukemia $(n=25 ; 2.5 \%)$, secondary myelofibrosis $(\mathrm{n}=40 ; 4.0 \%)$, or both $(\mathrm{n}=59 ; 5.8 \%)$ was detected in only a small number of patients during the study period, we could not assess the impact of $\mathrm{HU}$ on the development of these secondary malignancies. Another limitation is the need to exclude a large number of patients without continuous Medicare coverage and HMO participants, limiting the number of analyzed patients to 1,010 of 3,474 (29\%), although the excluded patients were comparable to those who were included in terms of age, sex, and race. Lastly, our study is observational in design and may be subject to possible confounding caused by unobserved factors. Although we controlled for many potential confounders, including sociodemographic characteristics, disability status, and a proxy measure for access to the healthcare system, it is possible that some residual confounding might have remained.

\section{Conclusions}

This study confirms the clinical benefits of HU in the management of older patients with ET and highlights the fact that this treatment is underused. A large number of patients continue to have disease-related complications. In addition to better implementation of current guidelines, further basic and translational studies are needed to improve patient outcomes.

Submitted June 28, 2019; accepted for publication October 11, 2019.

Author contributions: Funding acquisition: Podoltsev. Study concept: Podoltsev, Zhu, Zeidan, R. Wang, Ma. Formal analysis: Zhu. Methodology: R. Wang. Manuscript preparation: Podoltsev, Zhu, Ma. Manuscript review and editing: All authors.

Disclosures: Dr. Podoltsev has disclosed that he was a consultant for and received honoraria from Alexion and Pfizer, and received research support from Boehringer Ingelheim, Astellas Pharma, Daiichi Sankyo, Sunesis Pharmaceuticals, Celator, Pfizer, Astex Pharmaceuticals, CTI BioPharma, Celgene, Genentech, and LAM Therapeutics. Dr. Zhu has disclosed that she was a consultant for and received honoraria from AbbVie, Otsuka, Pfizer, Gilead, Celgene, Ariad, Incyte, Agios, Novartis, Takeda, Daiichi Sankyo, and Boehringer Ingelheim; was on the speakers bureau for Takeda; and received grant/research support from Celgene, Pfizer, Incyte, ADC Therapeutics, Medimmune, Takeda, AbbVie, and Boehringer Ingelheim. Dr. Davidoff has disclosed that she has received grant/research support from Celgene. Dr. Huntington has disclosed that he has received honoraria from Pharmacyclics and was a consultant for Celgene and Janssen. Dr. Gore has disclosed that he was consultant for and has received research support from Celgene. Dr. Ma has disclosed that she was a consultant for Celgene and Incyte and has received research support from Celgene. The remaining authors have disclosed that they have not received any financial considerations from any person or organization to support the preparation, analysis, results, or discussion of this article.

Funding: This research was supported by the Frederick A. Deluca Foundation.

Disclaimer: The collection of the California cancer incidence data used in this study was supported by the California Department of Public Health as part of the statewide cancer reporting program mandated by California Health and Safety Code Section 103885; the NCl's SEER program under contract N01-PC-35136 awarded to the Northern California Cancer Center, contract N01-PC-35139 awarded to the University of Southern California, and contract N02-PC-15105 awarded to the Public Health Institute; and the CDC's National Program of Cancer Registries, under agreement \#U55/ CCR921930-02 awarded to the Public Health Institute. The ideas and opinions expressed herein are those of the authors and endorsement by the State of California, Department of Public Health, the $\mathrm{NCl}$, and the CDC or their Contractors and Subcontractors is not intended nor should be inferred. The authors acknowledge the efforts of the Applied Research Program, $\mathrm{NCl}$; the Office of Research, Development and Information, CMS; Information Management Services, Inc.; and the SEER Program tumor registries in the creation of the SEER-Medicare database. The interpretation and reporting of the SEER-Medicare data are the sole responsibility of the authors.

Correspondence: Nikolai A. Podoltsev, MD, PhD, Department of Internal Medicine, Section of Hematology, Yale School of Medicine, 37 College Street New Haven, CT 06510. Email: nikolai.podoltsev@yale.edu

\section{References}

1. Srour SA, Devesa SS, Morton LM, et al. Incidence and patient survival of myeloproliferative neoplasms and myelodysplastic/myeloproliferative neoplasms in the United States, 2001-12. Br J Haematol 2016;174:382-396.

2. Tefferi A, Guglielmelli P, Larson DR, et al. Long-term survival and blast transformation in molecularly annotated essential thrombocythemia, polycythemia vera, and myelofibrosis. Blood 2014;124:2507-2513, quiz 2615

3. Tefferi A, Barbui T. Polycythemia vera and essential thrombocythemia: 2017 update on diagnosis, risk-stratification, and management. Am J Hematol 2017;92:94-108.

4. Hultcrantz M, Björkholm M, Dickman PW, et al. Risk for arterial and venous thrombosis in patients with myeloproliferative neoplasms: a populationbased cohort study. Ann Intern Med 2018;168:317-325.

5. Barbui T, Barosi G, Birgegard G, et al. Philadelphia-negative classical myeloproliferative neoplasms: critical concepts and management recommendations from European LeukemiaNet. J Clin Oncol 2011;29: 761-770.

6. Mesa RA, Jamieson C, Bhatia R, et al. NCCN Guidelines Insights: myeloproliferative neoplasms, version 2.2018. J Natl Compr Canc Netw 2017;15:1193-1207.

7. Barbui T, Tefferi A, Vannucchi AM, et al. Philadelphia chromosomenegative classical myeloproliferative neoplasms: revised management recommendations from European LeukemiaNet. Leukemia 2018;32: 1057-1069.

8. Mascarenhas J, Mesa R, Prchal J, et al. Optimal therapy for polycythemia vera and essential thrombocythemia can only be determined by the completion of randomized clinical trials. Haematologica 2014;99: 945-949.

9. Cortelazzo S, Finazzi G, Ruggeri M, et al. Hydroxyurea for patients with essential thrombocythemia and a high risk of thrombosis. N Engl J Med 1995;332:1132-1136.

10. Harrison CN, Campbell PJ, Buck G, et al. Hydroxyurea compared with anagrelide in high-risk essential thrombocythemia. N Engl J Med 2005; 353:33-45. 
11. Gisslinger $\mathrm{H}$, Gotic M, Holowiecki J, et al. Anagrelide compared with hydroxyurea in WHO-classified essential thrombocythemia: the ANAHYDRET study, a randomized controlled trial. Blood 2013;121:1720-1728

12. Spivak JL. Myeloproliferative neoplasms. N Engl J Med 2017;377: 895-896.

13. Warren JL, Klabunde CN, Schrag D, et al. Overview of the SEER-Medicare data: content, research applications, and generalizability to the United States elderly population. Med Care 2002;40(8 Suppl):IV-3-18.

14. Zuo J, Curtis LM, Yao X, et al. Glucocorticoid receptor expression in the postnatal rat cochlea. Hear Res 1995;87:220-227.

15. Price GL, Davis KL, Karve $\mathrm{S}$, et al. Survival patterns in United States (US) Medicare enrollees with non-CML myeloproliferative neoplasms (MPN). PLoS One 2014;9:e90299.

16. Brière JB. Essential thrombocythemia. Orphanet J Rare Dis 2007;2:3

17. Kind S, Virnig B, McBean AM. SEER-Medicare: Defining the Date of Diagnosis \& Treatment. Available at: https://healthcaredelivery.cancer. gov/seermedicare/considerations/date.html. Accessed September 14, 2018.

18. Hollenbeck BK, Ye Z, Dunn RL, et al. Provider treatment intensity and outcomes for patients with early-stage bladder cancer. J Natl Cancer Inst 2009;101:571-580.

19. Healy MA, Yin HY, Reddy RM, Wong SL. Use of positron emission tomography to detect recurrence and associations with survival in patients with lung and esophageal cancers. J Natl Cancer Inst 2016;108:djv429.
20. Raebel MA, Schmittdiel J, Karter AJ, et al. Standardizing terminology and definitions of medication adherence and persistence in research employing electronic databases. Med Care 2013;51(8 Suppl 3): S11-21.

21. Gupta A, Long JB, Chen J, et al. Risk of vascular toxicity with platinum based chemotherapy in elderly patients with bladder cancer. J Urol 2016 195:33-40.

22. Davidoff AJ, Zuckerman IH, Pandya N, et al. A novel approach to improve health status measurement in observational claims-based studies of cancer treatment and outcomes. J Geriatr Oncol 2013;4:157-165.

23. Klabunde CN, Potosky AL, Legler JM, et al. Development of a comorbidity index using physician claims data. J Clin Epidemiol 2000;53: 1258-1267.

24. Schoenfeld D. Partial residuals for the proportional hazards regression model. Biometrika 1982;69:239-241.

25. Previtali E, Bucciarelli P, Passamonti SM, et al. Risk factors for venous and arterial thrombosis. Blood Transfus 2011;9:120-138.

26. Vannucchi AM, Guglielmelli P. What are the current treatment approaches for patients with polycythemia vera and essential thrombocythemia? Hematology Am Soc Hematol Educ Program 2017;2017:480-488.

27. Barbui T, Vannucchi AM, Buxhofer-Ausch V, et al. Practice-relevant revision of IPSET-thrombosis based on 1019 patients with WHO-defined essential thrombocythemia. Blood Cancer J 2015;5:e369.
NCCN National Comprehensive NCCN Cancer Network ${ }^{\circledast}$

Live
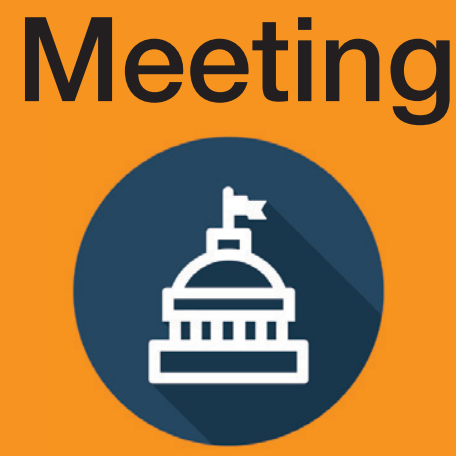

SAVE THE DATE
NCCN ONCOLOGY POLICY SUMMIT:

\section{The State of Cancer Care in America: The Impact of State Policy on Access to High- Quality Cancer Care}

\author{
Thursday, June 27, 2019 | Washington, DC
}

The summit will explore the increasing role of state authorities in health policy decision-making and implications for patient access to high-quality cancer care. Major themes of the summit will include the current state regulatory environment governing insurance marketplaces and benefit design, variability across federal and state-level health care coverage landscapes, and best practices for policy makers, providers, and patient advocates to protect patient access to highquality cancer care within shifting legislative and regulatory environments.

\section{Visit NCCN.org/policy to learn more.}

